\title{
Ultra high speed full adder for biomedical applications
}

\author{
Basavoju Harish', M. S. S. Rukmini² \\ ${ }^{1}$ Department of Electronics and Communication Engineering, Vignan's Lara Institute of Technology \& Science, India \\ ${ }^{2}$ Department of Electronics and Communication Engineering, Vignan's University, India
}

\begin{tabular}{l}
\hline \hline Article Info \\
\hline Article history: \\
Received Dec 10, 2020 \\
Revised Jan 10, 2021 \\
Accepted Jan 20, 2021 \\
\hline
\end{tabular}

\section{Keywords:}

10 transistor

CMOS

Full adder

High Speed

PDP

\begin{abstract}
In the field of biomedical engineering high performance CPU for digital signal processing plays a significant role. Frequency efficient circuit is a paramount requirement for the portable digital devices employing various digital processors. In this work a novel high speed one-bit 10T full adder with complemented output was described. The circuit was constructed with XOR gates which were built using two CMOS transistors. The XOR gate was constructed using $2 \mathrm{~T}$ multiplexer circuit style. It was observed that power consumption of the designed circuit at $180 \mathrm{~nm}$ with supply voltage $1.8 \mathrm{~V}$ is $183.6 \mathrm{uW}$ and delay was 1.809 ps whereas power consumption at $90 \mathrm{~nm}$ with supply voltage $1.2 \mathrm{~V}$ is $25.74 \mathrm{uW}$ and delay was $8.245 \mathrm{ps}$. The observed Power Delay Product (PDP) in 180nm (at supply voltage 1.8V) is 0.33 and in $90 \mathrm{~nm}$ (at supply voltage $1.2 \mathrm{~V}$ ) is 0.212 . The work was extended by implementing a 32-bit Ripple Carry Adder (RCA) and was found that the delay at $180 \mathrm{~nm}$ is $93.7 \mathrm{ps}$ and at $90 \mathrm{~nm}$ is $198 \mathrm{ps}$. The results were drawn at $180 \mathrm{~nm}$ and also $90 \mathrm{~nm}$ technology using CAD tool. The results say that the present work offered significant enhancement in speed and PDP compared with existing designs.
\end{abstract}

This is an open access article under the CC BY-SA license.

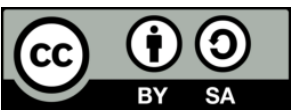

\section{Corresponding Author:}

Basavoju Harish

Department of Electronics and Communication Engineering

Vignan's Lara Institute of Technology \& Science

Guntur, India

Email:17harish@gmail.com

\section{INTRODUCTION}

Advancement in VLSI technology given path to immense development in portable digital equipments like mobile handsets, tablets, PDA's etc. The compact sizes, low power, high speed of operations are the key features of these applications. However still research had been going on to improve the performances of the applications. Every time it is a challenge for the VLSI engineers to bring tradeoff among the design entities like power consumption, delay and area of VLSI chips. Since, addition is basic operation that is extensively used in most of the processors, this module plays important role in arithmetic operations like addition, multiplication, division and address formation [1,2]. The power delay product (PDP) of the adder affects the total performance of the system. Considering this fact, the full adders are to be designed with high speed and less power [3].

Any digital processor consists of a full adder circuit at its initial levels of architecture. The full adder designs has the effect on the all the operations of processors like DSP architectures and microprocessors. The existing 28T full adder was designed using CMOS technology which consists of 28 transistors [4]. The demerits of 28 transistors CMOS full adder was its need of buffers and its high impedance and the merit is robustness against size of the transistors and scaling of voltage levels [5]. The output swing in complementary pass transistor (CPL) style full adder (32 transistors) was good but cannot be used for low 
power circuits $[6,7]$. The demerit of output degradation in CPL was eliminated in transmission gate adder (20 transistors). In the proposed circuit the full adder was constructed using only 10 transistors which promise full swing voltage levels at its outputs [8].The proposed circuit produced complemented sum and carry outputs. It used basic building blocks where each block consists of 2 transistor multiplexer circuit which produced XOR output as shown in Figure 2(a). The sum and carry outputs generated were given to the inverter circuits to produce full swing complemented sum and carry outputs [9-12]. The main aim of the proposed design was to show efficiency in the delay, PDP and number of transistors compared to other existing full adders. The proposed design was implemented by varying the width of the transistors in order to achieve full voltage swing at 90 and 180nm technology using SPICE tool as shown in Table 1. The propagation delay of the proposed circuit was $8.245 \mathrm{ps}$ and the power consumption was $25.74 \mathrm{uW}$ at $90 \mathrm{~nm}$ whereas propagation delay was $1.809 \mathrm{ps}$ and power consumption was $183.6 \mathrm{uW}$ at $180 \mathrm{~nm}$ technology as shown in Table 2 and Table 3.

\section{RESEARCH METHOD}

The proposed design consists of five blocks: module 1, 2, 3, 4 \& 5. Module 1, $2 \& 3$ consists of 2 transistor multiplexer circuit which produced XOR output as shown in Figure 1. Module 1, $2 \& 4$ generates the complemented sum and module $3 \& 5$ generates complemented carry. The width of the transistors in each module was varied such that the design entities like delay, area and PDP were optimized. The full logic swing of the output levels was obtained by varying the width of the transistors in the modules and placing the inverters at the outputs. These inverters will restore the logic levels resulted in complemented outputs. The carry output was constructed using 2 transistor multiplexer circuit only [13-15]. This carry output circuit consists of MP4 \& MN4; the output of this carry module was connected to inverter to achieve complemented carry. The inverter connected at the output produces full swing logic levels.

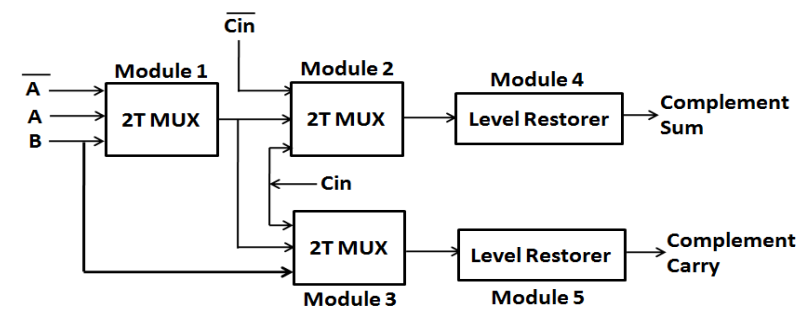

Figure 1. Block diagram of the proposed full adder design

\section{CIRCUIT OPERATION}

The module 1 consists of 2T XOR circuit which produced XOR output as shown in Figure 2(a). This 2T XOR (module 1) circuit consists of MP1 (PMOS) \& MN1 (NMOS) transistors. But there was a voltage degradation problem in it. To overcome the problem the width of the transistors MP1 \& MN1 are varied. Module 2 consists of 2T XOR where inputs were Cin and XOR output of module 1 . The module 2 consists of MP2 \& MN2 transistors whose channel widths were also varied as shown in Figure 2(b) [16, 17].

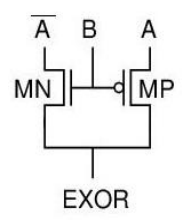

(a)

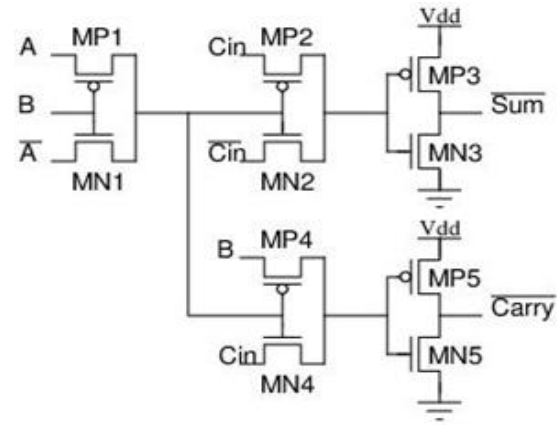

(b)

Figure 2. (a) 2T multiplexer circuit; (b) Proposed 10T fulladder 
The sum output of module 2 was applied to inverter to give complemented sum output which has full swing voltage level. Module 3 also consists of 2T XOR whose inputs were Cin and B applied to MP4 \& MN4 transistors where channel widths were also varied. The output of the module 3 was given to inverter which yielded complemented carry.

\section{PERFORMANCE OF THE PROPOSED DESIGN}

The proposed full adder as shown in Figure 2(b) was designed at 90 and 180nm technology. The design entities like transistor count, average power and delay was compared with previous designs [18, 19]. It was observed that in this design the propagation delay was reduced by less the number of transistors in the circuit and also due to with of the transistors as shown in Table 1.

Full voltage swing was obtained by connecting the outputs to the voltage restoration circuit (inverter).The proposed adder was compared with other adders. The results were compared by simulating all the adders in 90 and 180nm technology. The performance parameters like PDP, power and operating speed with the supply voltage $1.8 \mathrm{~V}$ and $1.2 \mathrm{~V}$ are drawn at $180 \mathrm{~nm}$ and $90 \mathrm{~nm}$ respectively and compared with existing designs. The comparisons are shown in the Tables 2 and Table 3.

Table 1. Transistor channel widths of proposed full adder

\begin{tabular}{ccc}
\hline Transistor Name & $\begin{array}{c}\text { Width } \\
\text { (180nm Technology) }\end{array}$ & $\begin{array}{c}\text { Width } \\
\text { (90nm Technology) }\end{array}$ \\
\hline MN1 & 200 & 120 \\
MN2 & 200 & 120 \\
MN3 & 200 & 120 \\
MN4 & 200 & 120 \\
MN5 & 200 & 120 \\
MP1 & 200 & 120 \\
MP2 & 200 & 120 \\
MP3 & 200 & 120 \\
MP4 & 200 & 120 \\
MP5 & 200 & 120 \\
\hline
\end{tabular}

\section{CALCULATION OF PROPAGATION DELAY AND POWER DISSIPATION}

The propagation delay in an adder is governed by the overall speed of the entire arithmetic unit of the processors or in other words overall speed of the design can be calculated using propagation delay. In this design the carry was generated using $2 \mathrm{~T}$ multiplexer logic circuit which acted like an XOR gate. The carry inputs (Cin) were given to this XOR gate (ie., module 2). To overcome the problem of voltage swing at the output the carry output was given to the inverter circuit which acted as level restorer. In this design the carry input was propagated through only one module ie., module 2 which acted as XOR gate as said above and hence leads to very less propagation delay [20]. However the widths of the transistors at $180 \mathrm{~nm}$ and $90 \mathrm{~nm}$ technology being changed to reduce the propagation delay [21].

Power consumption in VLSI circuits is due to switching of the transistors and short circuits.

The total power is given as

Ptotal $=$ Vdd. Fclk . $\sum$ iVswing.Cload.Pi + Vdd. $\sum$ ilsc + Vdd.Il

Where Vdd = supply voltage,

Vswing = voltage swing at the output

Cload =i load capacitance

Fclk = frequency of operation

$\mathrm{Isc}=$ short circuit current

$\mathrm{Il}=$ leakage current.

The average power consumption was calculated for the proposed design at $180 \mathrm{~nm}$ and $90 \mathrm{~nm}$ technologies. The supply voltage at $180 \mathrm{~nm}$ and $90 \mathrm{~nm}$ technologies was $1.8 \mathrm{v}$ and $1.2 \mathrm{v}$ respectively. The widths of the transistors in the proposed design at $180 \mathrm{~nm}$ and $90 \mathrm{~nm}$ technologies were $200 \mathrm{~nm}$ and $120 \mathrm{~nm}$ respectively. The power of the design at two different technologies was given in the Tables 2 and Table 3. It was observed that average power consumption of the design at $90 \mathrm{~nm}$ was less when compared to $180 \mathrm{~nm}$ technology $[22,23]$. 
Table 2. Simulation results at $180 \mathrm{~nm}$ technology

\begin{tabular}{cccccc}
\hline Design & Avg Power $(\mathrm{uW})$ & Delay $(\mathrm{ns})$ & PDP $(\mathrm{fJ})$ & Transistor Count & Reference \\
\hline CMOS & 6.2199 & 292.1 & 1.81683 & 28 & {$[4,18]$} \\
Mirror & 6.0797 & 281.61 & 1.71210 & 28 & {$[18]$} \\
CPL & 7.7198 & 183.97 & 1.42022 & 32 & {$[5,6]$} \\
TFA & 8.2491 & 287.1 & 2.36831 & 16 & {$[21]$} \\
TGA & 8.4719 & 293.9 & 2.8989 & 20 & {$[7,8]$} \\
14T & 12.721 & 381.7 & 4.85587 & 14 & {$[10]$} \\
10T & 14.344 & 132.59 & 1.90206 & 10 & {$[24]$} \\
HPSC & 6.3798 & 273.7 & 1.74615 & 22 & {$[11]$} \\
Majority Based & 6.3227 & 185.4 & 1.17222 & -- & {$[23]$} \\
24T & 15.91 & 314.2 & 4.998 & 24 & {$[1]$} \\
FA_Hybrid & 5.978 & 252.3 & 1.508 & 24 & {$[2]$} \\
FA_DPL & 19.56 & 226.6 & 4.432 & 22 & {$[19]$} \\
FA_SR-CPL & 20.78 & 220.65 & 4.432 & 20 & {$[19]$} \\
CMOS \& TG & 4.1563 & 224 & 0.931 & 16 & {$[19]$} \\
Proposed & 183.6 & 0.001809 & 0.33 & 10 & Present \\
\hline
\end{tabular}

\section{IMPLEMENTATION OF 32-BIT RCA}

The work was extended by implementing a 32-bit RCA in 180 and $90 \mathrm{~nm}$ technologies. The sum and carry outputs of full adder in each stage depend on carry of previous stage which results in producing the final carry with more delay. In this circuit the delay was very less compared to other standard designs. Overall delay of the RCA adder can be minimized by implementing using the design. From the obtained results it is observed that the overall delay produced by the 32-bit RCA in 90nm technology (at $1.2 \mathrm{~V}$ supply voltage) is $198.8 \mathrm{ps}$ and in $180 \mathrm{~nm}$ technology (at $1.8 \mathrm{~V}$ supply voltage) is $93.7 \mathrm{ps}$. 32-bit fulladder using 1-bit full adder multiplexer circuit as shown in Figure 3.

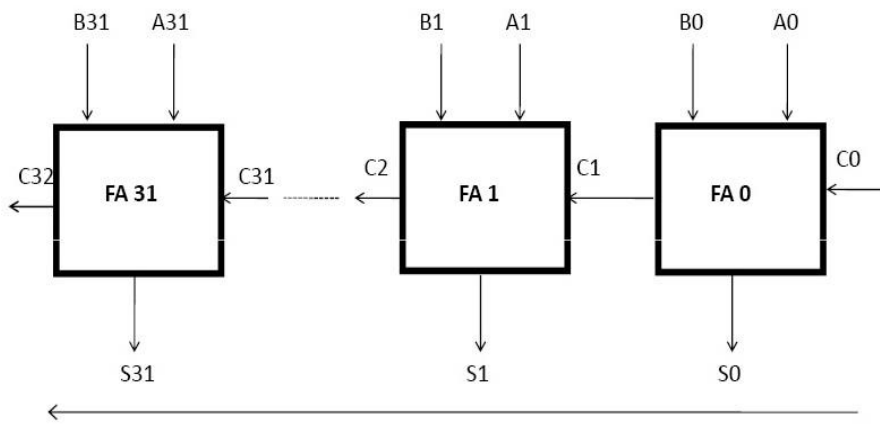

Carry Propagation Path

Figure 3. 32-bit fulladder using 1-bit full adder multiplexer circuit

Table 3. Simulation results at $90 \mathrm{~nm}$ technology

\begin{tabular}{|c|c|c|c|c|c|}
\hline Design & Avg Power (uW) & Delay (ns) & PDP (fJ) & Transistor Count & Reference \\
\hline CMOS & 1.5799 & 0.1269 & 0.2004 & 28 & {$[4,18]$} \\
\hline Mirror & 1.5701 & 0.1226 & 0.1924 & 28 & [18] \\
\hline CPL & 1.7598 & 0.0791 & 0.1392 & 32 & {$[5,6]$} \\
\hline TFA & 1.7363 & 0.3198 & 0.5552 & 16 & [21] \\
\hline TGA & 1.7619 & 0.2317 & 0.4082 & 20 & {$[7,8]$} \\
\hline $14 \mathrm{~T}$ & 3.3297 & 0.3389 & 1.1284 & 14 & [10] \\
\hline $10 \mathrm{~T}$ & --- & --- & --- & 10 & [24] \\
\hline HPSC & 1.56 & 0.2207 & 0.3442 & 22 & [11] \\
\hline Majority Based & 1.5751 & 0.0939 & 0.1479 & -- & [23] \\
\hline $24 \mathrm{~T}$ & 7.707 & 0.1406 & 1.0836 & 24 & [1] \\
\hline FA_Hybrid & 6.21 & 0.143 & 0.888 & 24 & [2] \\
\hline FA_DPL & 7.34 & 0.254 & 1.864 & 22 & [19] \\
\hline FA_SR-CPL & 7.4 & 0.167 & 1.235 & 20 & [19] \\
\hline CMOS \& TG & 1.17 & 0.0913 & 0.1074 & 16 & [19] \\
\hline Proposed & 25.74 & 0.008245 & 0.2122 & 10 & Present \\
\hline
\end{tabular}




\section{SIMULATION RESULTS}

In this work 15 different full adders were compared in terms of average power, delay and PDP .The simulation result of proposed 10T full adder was compared with different full adders viz 28T CMOS , 28T Mirror adder, 32T CPL, 16T TFA, 20T TGA, 14T Fulladder, 10T full adder, HPSC , majority based , $24 \mathrm{~T}$ Full adder, 24T FA_Hybrid , 22T FA_DPT, FA_SRCPL, 16T CMOS \& TG [24]. The simulation process was carried out by parametric analysis of the widths of the transistors so that proper voltage swing was obtained with less delay. The circuit design and simulation process was compromised in reducing the power consumption. However the delay of the circuit was greatly optimized when compared to all other designs. The power delay product (PDP) was also optimized due to very low propagation delay [25].

The proposed fulladder circuit was constructed using 10 transistors. The schematic of the fulladder was designed at $180 \mathrm{~nm}$ and $90 \mathrm{~nm}$ CMOS technologies using Cadence Virtuoso tool. Table 1 shows the widths of the transistors at $180 \mathrm{~nm}$ and $90 \mathrm{~nm}$ technologies. Tables 2 and 3 show the performance comparison regarding PDP, propagation delay and power consumption. From Table 2 it can be observed that the PDP was $35.4 \%$ efficient with the best design report at $180 \mathrm{~nm}$. Also from the Tables $2 \& 3$ the propagation delay was very less compared with all other circuits at $180 \mathrm{~nm} \& 90 \mathrm{~nm}$ technology as shown in Figures 5 and 6. The Figure 4 shows the comparison of PDP of various fulladders with the present work. In this paper a 32-bit RCA as shown in Figure 3 was implemented as an extension to the proposed work. In the implemented RCA only the delay was calculated. The performance evaluation was carried out at 180nm and 90nm technologies. The results shown that the overall delay produced by the 32-bit RCA in 90nm technology (at $1.2 \mathrm{~V}$ supply voltage) is $198.8 \mathrm{ps}$ and in $180 \mathrm{~nm}$ technology (at $1.8 \mathrm{~V}$ supply voltage) is $93.7 \mathrm{ps}$.

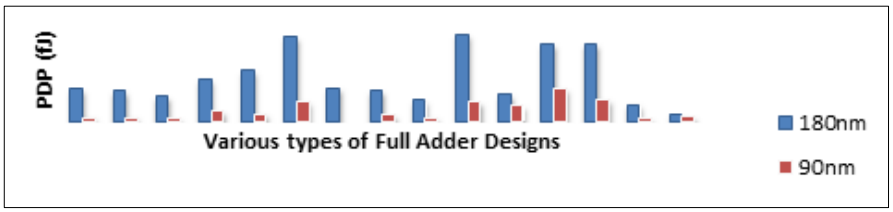

Figure 4. PDP of different full adder designs



Figure 5. Delay of different full adder designs at 90nm technology

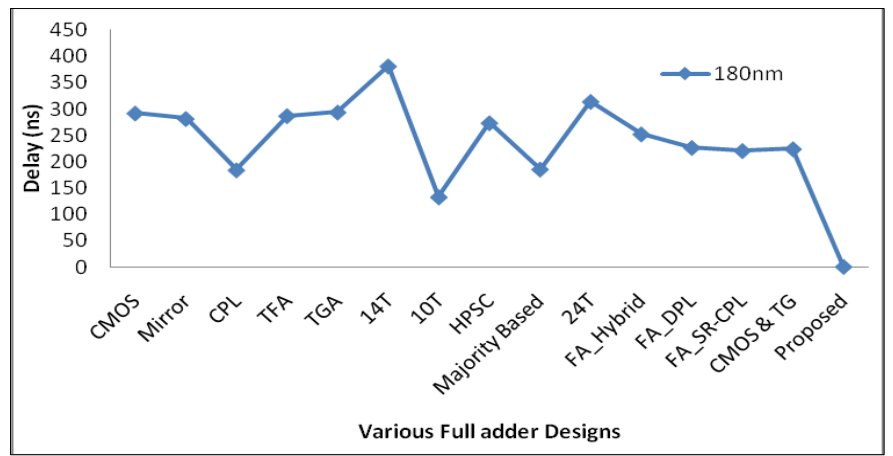

Figure 6. Comparison of delay of different full adder designs at 180nm technology 


\section{CONCLUSION}

Here a delay efficient adder was introduced. The design was carried out using SPICE tool at 90 and $180 \mathrm{~nm}$ technology. It was observed from the results that proposed circuit offers improved propagation delay \& PDP. From simulations it can be said that the adder circuit is $35.4 \%$ efficient in PDP wrt best design at $180 \mathrm{~nm}$ technology and $10.4 \%$ efficient in delay wrt best design at $90 \mathrm{~nm}$ technology. The circuit was further used to implement a 32-bit RCA. The propagation delay of 32-bit RCA at 100MHz in 90nm technology (at $1.2 \mathrm{~V}$ supply voltage) is $198.8 \mathrm{ps}$ and in $180 \mathrm{~nm}$ technology (at $1.8 \mathrm{~V}$ supply voltage) is $93.7 \mathrm{ps}$.

\section{REFERENCES}

[1] X. S. Li, et al., "Analysis and simplification of three-dimensional space vector PWM for three-phase four-leg inverters," IEEE Transactions on Industrial Electronics, vol. 58, pp. 450-464, Feb 2011.

[2] Tung, Shieh, "A low-power high-speed hybrid CMOS full adder for embedded system," IEEE Conference on Design Diagnostics E C Systems., vol. 13, Apr. 2007.

[3] Bayoumi, "Design of robust, energy efficient full adders for deep-submicrometer design using hybrid-CMOS logic style," IEEE Transactions (VLSI)., vol. 14, Dec. 2006.

[4] Weste, CMOS VLSI design, 3rd ed. Delhi, India: Pearson Publishers, 2006.

[5] J. M. Rabaey, Digital integrated circuits, Pearson Publishers, 2003.

[6] D. R krishnan, "Low-voltage low-power CMOS full adder," IEEE Circuits Devices Systems., vol. 148, Feb. 2001.

[7] R. Zimmermann, "Low-power logic styles: CMOS versus pass-transistor logic," Journal of Solid State Circuits, IEEE, vol. 32, Jul. 1997.

[8] Chang, "A review of 0.18 - $\mu \mathrm{m}$ full adder performances for tree structured arithmetic circuits," IEEE Transactions on VLSI, vol. 13, Jun. 2005.

[9] M. Shams and M. A, "Performance analysis of low-power 1-bit CMOS full adder cells," IEEE Transactions on VLSI, vol. 10, Feb. 2002.

[10] Baez, "Hybrid adders for high-speed arithmetic circuits: A comparison," Proceedings of 7th IEEE International Conference Electrical Eng., NM, USA, Sep. 2010.

[11] Vesterbacka, "A 14-transistor CMOS full adder with full voltageswing nodes," in Proceedings IEEE Workshop on Signal Process, Taiwan, Oct. 1999.

[12] Zhang, "Novel hybrid pass logic with static CMOS output drive full-adder cell," in Procedeeings of International Symposium Circuits, May 2003.

[13] Wairya, "Design analysis of XOR (4T) based low voltage CMOS full adder circuit," in Proceedings of IEEE Nirma Univ, (NUiCONE), Dec. 2011.

[14] Goel, "Novel design methodology for high-performance XOR-XNOR circuit design," in Proceedings of. 16th Symposium Integration Circuits, Sep. 2003.

[15] Wang, "New efficient designs for XOR and XNOR functions on the transistor level," IEEE Journal of Solid State Circuits, vol. 29, Jul. 1994.

[16] Prashanth, "Architecture of adders based on speed, area and power dissipation," in Proceedings of World Congress Information Communication Technology, Dec. 2011.

[17] B. Harish, K. Sivani and M. S. S. Rukmini, "Design and performance comparison among various types of adder topologies," 2019 3rd International Conference on Computing Methodologies and Communication (ICCMC), Erode, India, 2019, pp. 725-730, doi: 10.1109/ICCMC.2019.8819779.

[18] B. Harish, K. Sivani and M. S. S. Rukmini, "Design of high speed efficient parallel prefix brent-kung adder architecture," Journal of Green Engineering, vol. 10, no. 7, pp. 3508-3519, Jul. 2020.

[19] B.Harish, K. Sivani and M. S. S. Rukmini, "Implementation of frequency efficient multiplexer based CORDIC on FPGA," International Journal of Advanced Science and Technology, vol. 29, no. 05, pp. 753-762, Apr. 2020.

[20] B. Harish, K. Sivani and M. S. S. Rukmini, "Performance comparison of various CMOS full adders," 2017 International Conference on Energy, Communication, Data Analytics and Soft Computing (ICECDS), Chennai, 2017, pp. 3789-3792, doi: 10.1109/ICECDS.2017.8390172.

[21] P. Bajpai, P. Mittal, A. Rana and B. Aneja, "Performance analysis of a low power high speed full adder," in 2017 2nd International Conference on Telecommunication and Networks (TEL-NET), Noida, 2017, pp. 1-5, doi: 10.1109/TEL-NET.2017.8343543.

[22] V. Agarawal, R. Shrivastava and S. Akashe, "Performance and analysis of 10T Full Adder using MTCMOS technique," in 2015 International Conference on Communication Networks (ICCN), Gwalior, 2015, pp. 290-295, doi: 10.1109/ICCN.2015.56.

[23] S. S. Purohit, S. R. Chalamalasetti, M. Margala and W. A. Vanderbauwhede, "Design and evaluation of highperformance processing elements for reconfigurable systems," in IEEE Transactions on Very Large Scale Integration (VLSI) Systems, vol. 21, no. 10, pp. 1915-1927, Oct. 2013, doi: 10.1109/TVLSI.2012.2220868.

[24] R. Gupta, Satya Prakash Pandey and S. Akashe, "Comparative analysis and optimization of active power and delay of 1-bit full adder at $45 \mathrm{~nm}$ technology," 2013 Students Conference on Engineering and Systems (SCES), Allahabad, 2013, pp. 1-5, doi: 10.1109/SCES.2013.6547556.

[25] M. Yadav, A. Gupta and S. Rai, "A study and comparative analysis of low power hybrid-CMOS 1-bit full adders in deep-submicron technology," in 2017 4th International Conference on Power, Control \& Embedded Systems (ICPCES), Allahabad, 2017, pp. 1-7, doi: 10.1109/ICPCES.2017.8117618. 


\section{BIOGRAPHIES OF AUTHORS}

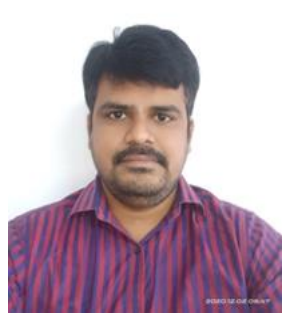

Basavoju Harish received the B.Tech degree from Kakatiya University, India in 2006 and M.Tech degree in VLSI Design from Anurag Engineering College, India. He is working as Assistant Professor at Vignan's Lara Institute of Technology \& Science, India and pursuing his Ph.D at Vignan's Foundation for Science, Technology \& Research University, Guntur, India. His current research interest includes Fast Adder Architectures for Digital Signal Processors.

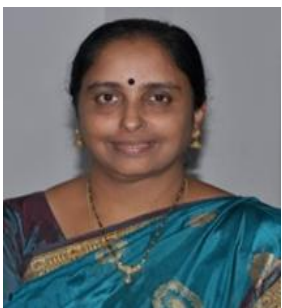

Dr. M.S.S.Rukmini received B.E in Electronics and Communications and M.E in Applied Electronics under the affiliation of Madras University. She also received Ph.D degree in Electronics Science with Department of Computer science from Berhampur University, Orissa, India. Her research interests include wireless Communications, Embedded Systems and Internet of Things .She is now working as Professor in the department of Electronics and Communication Engineering from Vignan's Foundation for Science, Technology \& Research University, Andhra Pradesh, India. 